\title{
Comparison of student and instructor reasons for using computation
}

\author{
W. Brian Lane and Cortney Headley \\ Department of Physics, University of North Florida, 1 UNF Drive, Jacksonville, FL, 32224
}

\begin{abstract}
Attending to students' motivation to adopt computation as a physics practice is essential in effectively integrating computation into physics education. Within the Communities of Practice (COP) framework, addressing this motivational need involves inducting students into the community's sense of joint enterprise, which includes physicists' motivating reasons for adopting computational practices. We used the COP framework to interview students and instructors in the semester after they completed a set of computationally integrated upper division physics courses. This timing allowed us to assess students' perceptions of computation after they were no longer required to engage in computation in their coursework. This article discusses the reasons for using computation in physics identified by these students and instructors. We find that the students saw computation as a normative physics practice, and that they identified reasons for using computation that are consistent with those held by their instructors and the broader physics community. However, we also observe differences in the ways that these instructors and students articulated these reasons: The instructors drew on their research experience with computation, while the students drew on experience from coursework; each student tended to focus their overall discussion on a smaller subset of reasons than the instructors did; and students tended to discuss reasons in isolation, while instructors tended to interweave multiple reasons. We interpret these differences based on the students' positions and trajectories within the physics community.
\end{abstract}




\section{INTRODUCTION}

\section{A. Physics Education Research into the Use of Computation}

The use of computers to study, solve, and visualize physics problems is valuable in undergraduate physics education. Computation helps prepare students for careers in STEM research and industry [1-5] and enables students to pursue creative solutions [6, 7], engage in sense-making [8-10], and test model-based predictions in analytically intractable problems $[2,7,11-16]$. Although computation is widely adopted in university physics programs [17] and there is a consensus that computational experience is beneficial for students, research is needed to address challenges that arise when integrating computation, such as student motivation $[18,19]$ and "making room" in the curriculum [2, 14, 19]. Addressing these needs requires a clear and persuasive vision of why computation is beneficial to physics education and practice.

A concern often voiced among computationally minded physics educators is that, while we can expose students to computational methods and skills, they don't necessarily come to see these methods and skills as practices regularly used by physicists [20]. As such, it can be challenging to generate student buy-in for computation [21], and students might compartmentalize their computational learning to a particular course and not transfer that learning to other contexts [22].

\section{B. Computation as a Practice of the Physics Community}

To study this issue, we consider computation through the Communities of Practice (COP) framework [23-26], which is particularly useful when studying computation in physics education $[3,5,27,28]$. The community of physicists can be considered on the scale of a research group, a class, a department, or a discipline. Each member of this community is located at a different stage of development along a unique trajectory, with expert-like members at the center and novicelike members at the periphery of the community [26]. These members use computation (along with experiment and theory) as part of an ensemble of interconnected practices to pursue a shared set of goals [20]. The combination of practices that this community employs and the normative goals that its members pursue comprise the community's sense of joint enterprise, or "what counts" as doing physics [26].

From the perspective of this framework, a primary goal of computationally integrated physics education is to offer students a local representation of the global physics community's sense of joint enterprise [29]. This local representation helps students adopt computation in general as a practice and begin to adopt more specific practices of computational thinking [21, 30], expanding their own scope of what counts as doing physics on their way toward the center of the COP $[25,31,32]$. This adoption is often a rough and non-linear process $[26,33]$, which we focus on in our study.

\section{Our Study}

In this study, we consider the question, "To what degree have students adopted the shared practice of computation after encountering computation in a physics course?" This question emphasizes the persistence of students' perceptions of computation after they are no longer required to engage in it as part of their coursework. We find this question to be impactful as students head into industry and graduate school, where employers and supervisors might assume that students have already learned and adopted some computational practices along their trajectory $[5,27,28]$. We investigated this question through interviews of students and instructors who recently completed or taught computationally integrated physics courses. This paper focuses on the reasons that these students and instructors identified for using computation in physics.

Section II establishes the academic context and methodology of our study. Section III explores the reasons that students and instructors identified for using computation as a physics practice. Finally, Section IV compares the ways in which they discussed these reasons.

\section{CONTEXT AND METHODOLOGY}

We carried out this study in the physics program at a midsized regional state university that primarily serves undergraduates. This program graduates 10-20 physics bachelors each year and emphasizes research opportunities for undergraduates. In fall 2020, three faculty members (including this paper's first author) integrated regularly occurring computational assignments into three upper-division physics courses (Astrophysics I, Mathematical Physics, and Modern Physics). These three courses primarily serve physics majors but occasionally enroll students majoring in other STEM fields. A handful of students were enrolled in more than one of these courses, such that they experienced computation in multiple physics courses in the same semester.

The students in these courses had varying degrees of prior experience with programming. Some had taken an introductory programming course offered by the computing sciences department, while most had never encountered programming beyond using spreadsheets in introductory physics labs.

The instructors had all participated in computation-based professional development through the Partnership for Integration of Computation into Undergraduate Physics [34] and designed their computational activities to orient students to relevant programming tasks before introducing physics applications. The three instructors discussed their plans and progress throughout the semester. Following departmental norms, computational activities included minimally working programs [35-38] written in Jupyter notebook (Python) on a university server. Therefore, students enrolled in multiple computationally integrated courses had similar learning experiences across their coursework. Because this common set 
of students had similar experiences, we define the COP of our study as the physics majors and faculty within this physics department, which we view as a local representation of the broader physics community [29, 39].

We conducted semistructured interviews in the spring 2021 semester, two to four months after students completed these computationally integrated physics courses. Students and instructors were recruited in January through March 2021 from the fall 2020 class rosters, with interviews conducted in February through April 2021. Five students (Chrissie, Guy, Harrison, Jose, and Paul) and all three instructors (Jack, Lance, and Marlee) agreed to participate. The students' course enrollments and prior experiences with programming are listed in Table I. As one of these instructors is the first author on this paper, all instructor interviews were conducted by the second author. The authors shared responsibility for analyzing interview transcripts. The first author reviewed and coded the other instructors' interviews first and then used his own transcript primarily to confirm themes already identified. We acknowledge that this timeline places the computationally integrated courses and the interviews in the midst of the COVID-19 pandemic. As such, the three courses featured split remote/on-site teaching formats, and the interviews took place over video conference.

We sent student subjects a brief pre-interview survey about their career goals and experiences with programming. We also asked student and instructor subjects to provide a pseudonym to help maintain confidentiality during the interview. All five students reported that their spring 2021 courses did not heavily integrate computation, so we believe their interview responses are largely based on their fall 2020 experiences with computation.

We analyzed interview transcripts using the method of constant comparison [40], in which each author independently coded the transcripts to identify emergent themes that address our research questions. We revisited our theme definitions and coding criteria throughout the process and compared our themes and coding incidents after completing the full set of transcripts. This comparison produced an agreed-upon set of themes and coding criteria, which we used to review the transcripts once more to finalize the coding and select excerpts for this paper.

TABLE I. Summary of student interview subjects.

\begin{tabular}{lll}
\hline \hline Student & Physics Enrollments & Programming Background \\
\hline Chrissie & Math. Phys., Mod. Phys. & Prog. I \& II \\
Guy & Astro. I, Math. Phys. & Prog. I \\
Harrison & Math. Phys., Mod. Phys. & Prog. I \\
Jose & Math. Phys., Mod. Phys. & None \\
Paul & Math. Phys., Mod. Phys. & Prog. I \& II \\
\hline \hline
\end{tabular}

\section{INTERVIEW INSIGHTS ABOUT REASONS TO USE COMPUTATION}

These interviews reveal a wealth of insights into the students' and instructors' perceptions of computation. This preliminary report focuses on the reasons these subjects identified for using computation. By "reason," we mean a motivating advantage, benefit, necessity, or outcome achieved by using computational practices when studying or doing physics. For example, fitting a model equation to a data set is advantageously easier using a computer, while studying an analytically intractable problem is possible only through a computer's iterative application of an approximation scheme. This section outlines the reasons that students and instructors identified in these interviews. Quotes have been edited to remove pauses and repeated words, and emphasis has been added to highlight the subjects' reasoning.

\section{A. Student-Identified Reasons to use Computation}

Interview questions were designed to solicit students' thoughts on physics practices, and computation in particular. For example, the first interview question asked, "When you picture a physicist conducting research, what kinds of activities do you imagine them doing?" All five students included the use of computers in their answers to this question. The students did know that this interview would focus on the use of computation in physics, but we did not previously mention computation in the interview questions. This trend seems to indicate that the students saw computation as a normative part of the physics community's sense of joint enterprise. Subsequent interview questions elaborated on the use of computation, such as, "Why do you think physicists use computation in their research?", "What sort of computational skills, if any, do you think every physics student should learn about?", and "When you see your future self as a [career goal], how do you see yourself using computation, if at all?"

These students described professionally and personally important reasons for using computation. Collectively, the students identified many reasons for using computation consistent with those expressed by the physics community [1-16]: accuracy, aiding learning, conceptual and mathematical accessibility, data collection and analysis, efficiency, experimental control, manipulation of variables, personal applications, sense-making, simulation, and visualization.

For example, Guy discussed how computation "gave you a visualization of how the math worked... to see how the different formulas... came out... and it just gave a different view." Chrissie similarly highlighted visualization and connected it to sense-making, saying that she valued "being able to represent [stationary states] in a way that we could... digest more easily because it's not like we could create... something like an infinite square well... Through the code, you can... see like through a graph that this is what happens at this energy." Harrison focused his discussion on the efficiency afforded 
by computation over analytical problem-solving: "And being that it's a computer... it's done in an instant. It saves so much time and effort to review code instead of reviewing twenty-four pages of work that the computer could be doing in a nanosecond." Paul focused much of his discussion on using computation in the process of sense-making: "I kind of just started recognizing relationships and what I had to do... to get closer to the answer, rather than plugging something in and working on a calculation for an hour." Jose commented at length about sense-making when discussing how computation helped him in Modern Physics: "I think [computation] was helpful at understanding the kind of weird concepts... You have to almost think about... the various concepts in different ways, so that you can put them into a program... There was a radioactive decay assignment that was helpful because I had done a lab about decay and... I thought I had like a good understanding of it but in kind of putting in decay rates [in the program] that helped me understand more about what was going on... Putting it in computation, there are some questions asking about, 'What might happen if we change this?' and then you... change it and kind of see how that interacts..."

Additionally, two students described personal reasons for using computation, highlighting successful transfer of computational practices to new contexts [22]. Guy (who shared that he works as a lab manager), said, "I use computation at work every day, just to organize the sheer amount of chemicals that we have... Computation does kind of leak its way into daily life, whether you like it or not." Paul described his expectations that computation would be useful throughout his career: "I mean, it's so relevant. I feel like once I got comfortable at the job, if I'm doing something, you know, a thousand times, I'm just going to say, 'Oh, I wish I had a program for this' and I probably just go about making one."

Examining each interview in its entirety, we find that, while the students collectively identified various reasons for using computation, each student tended to focus their discussion on a much smaller subset of these reasons. Within interview segments (between questions from the interviewer), these students tended to discuss one or two reasons for using computation in relative isolation (as illustrated in the excerpts above).

\section{B. Instructor-Identified Reasons to use Computation}

The instructors we interviewed outlined a nearly identical set of reasons for using computation as a physics practice as the students did. In the context of teaching, these instructors highlighted the authenticity that computation affords. For example, Marlee expressed a goal to "get students comfortable with the idea of computation as a resource for understanding physics and math.” When discussing Astrophysics I, Jack contrasted computational assignments with analytical assignments on the basis of authenticity: "I've added computation in upper level courses for physics majors as part of an effort to give them more authentic research experiences in the classroom... I have [students] do analytical assignments like problem sets. These are shorter and generally they're more comfortable with that format. But I don't find them to be very authentic to... how the subject is practiced." Lance highlighted the value of students developing authentic computational skills that could be transferred to a professional context: "I want them to develop computational skills like debugging, like evaluating whether this particular algorithm is appropriate for the task at hand."

The instructors described their motivation to incorporate computation into their coursework based on their researchrelated uses of computation, and how this usage guides their decisions of how to integrate computation into their coursework. For example, Jack, who works with large data sets, said, "I think every physicist should be able to download a simple data set and at least construct a simple model, the kind of the basics of data science... That's, you know, how we can now trade data and check each other's results." When discussing Astrophysics I, Jack mentioned how computation enables his students to interact with authentic, professionally relevant data: "So one of the learning objectives [of Astrophysics I] is to apply knowledge to actual data... The computational assignments are really the only assignments that are directed towards that objective of interacting with the real data sets." When Marlee discussed his research, he described the value of using computation to obtain analytically intractable results and visualize those results: "Sometimes we use it to do calculations of, you know, physical processes that... cannot be calculated analytically. We use it for visualization and, you know, representation of the results of things that we've done." Later, Marleee revisited these same reasons when describing an example from his introductory calculus-based physics course: "This simulation... showed them the position vector, the velocity vector and the acceleration vector for a wheel as it was rolling along. And so this was an example that they sort of calculated by hand. They also record a video of a rolling wheel and then track the motion that way and then they have this computational model of the rolling wheel... So they got to work on it theoretically, they got to do an experimental measurement of it, and then they got to see how the computational model fit into there as well." When discussing his statistics-heavy use of computation in research, Lance focused on sense-making: "You can't just point to a set of data and say, 'This is significant.' The statistics is the impartial way of evaluating whether the thing you're looking at is significant..." Then, Lance revisited this reason when discussing computational skills he wants his students to develop: "I want them to see computational results and be able to tell, 'This region looks like it's reasonable, but this region where it's wiggling and going off to infinity is not reasonable." We argue that this connection between the instructors' research and educational practices highlights their central location with the physics COP: They are experienced with research-related uses of computation, and have an instructional goal of guiding students to adopt these uses as personally relevant. Their computationally integrated teaching mimics the COP's sense of joint enterprise. 
Finally, many of the instructors' interview segments chained together multiple reasons (underlined in square brackets below) for using computation. For example, when asked to describe how he uses computation in Mathematical Physics, Marlee said, "An example would be they're supposed to find the eigenvalues and eigenvectors for a matrix... They're sort of working out by hand... and then they're also sort of doing the calculations within, you know, using the computer as well to sort of see how they get to the same results and kind of see the utility of using a computer, especially for something that has a long hand calculation [efficiency]. But there are some times as well that we've just used the computer to visualize a more complicated calculation [visualization]... And then you can very quickly, just... look at it and say, 'Okay, yeah, this is doing what it's supposed to be doing' [sense-making]." When describing educational benefits of computation, Lance produced a similar chain: "[My students] are usually getting out either a graph or an animation or both [visualization]... And so I try to use that to produce something at the end that's going to engage my students in the actual analysis process [analysis]... and start to think about, 'What do I trust in these results? What seems sketchy in these results? How can I make these results better? Or how could I explore these results?' [sense-making] Further, you know, I want them to... produce something that gets them into the cycle of making tweaks to the code, examining the results and basically using it as a learning opportunity [learning]." Jack's interview featured similar chains, such as, "[Y]ou may have a system that mathematically, analytically the students are not capable of solving, but if it can be presented in a computational fashion, then it's trivial [mathematical accessibility] to run... the differential equations over and get a solution that they can visualize [visualization] that, you know, evolves in time dynamically or allows them to tweak parameters [manipulation] and explore how this system behaves."

\section{DISCUSSION AND CONCLUSION}

Here we contrast students' and instructors' discussions of reasons for using computation in physics. Students and instructors identified similar reasons for using computation in physics that align with reasons held by the broader physics community. We take this alignment to mean that the instructors have successfully initiated their students into the physics community's sense of joint enterprise related to computation. This observation lines up with similar findings that students tend to engage in computational thinking more actively during instructor intervention [21]. We recognize that, because of our small sample size, we cannot generalize this alignment to the entire population (especially since the students volunteered for these interviews), but we find that this learning experience was successful for these students in this regard. This finding is welcome in light of the negative evidence discussed in Section I A [20-22], and we speculate that the reinforce-

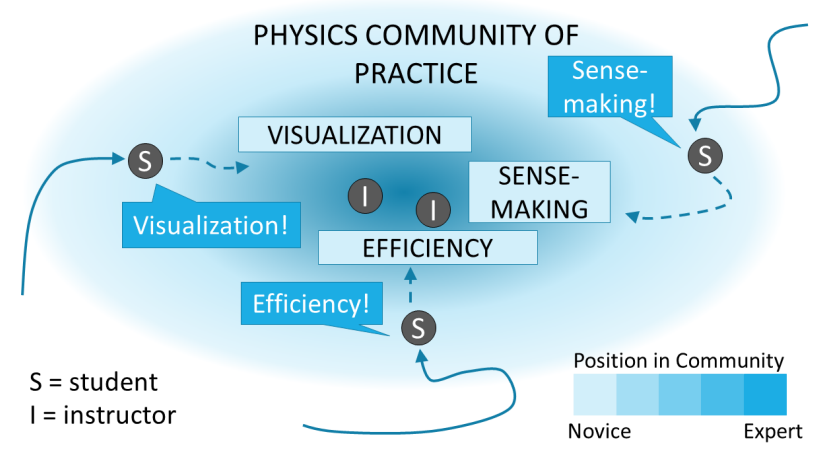

FIG. 1. The centrally located instructors (I) promote many reasons (rectangles) for using computation, while each peripherally located student (S) identifies most closely with a reason (speech bubble) based on their trajectory (curves).

ment of computational integration across concurrent courses helped support this development.

We also draw three key distinctions between the two groups: (1) The instructors drew on their research experience with computation, while the students drew on experience from class, which provided a view into the instructors' research experiences. (2) Each student tended to focus their overall discussion on a smaller subset of reasons for using computation, while instructors tended to discuss a breadth of reasons more equally. (3) Within each interview segment, each student tended to discuss one or two isolated reasons, while instructors tended to discuss multiple reasons within the same interview segment and weave those reasons together.

These differences suggest that the instructors view computation through a more holistic sense of joint enterprise from a more central position within the physics COP, while the students are still developing their sense of joint enterprise as they navigate toward the COP's center. We illustrate this model in Figure 1, in which students (S) navigate diverse trajectories (curves) toward the center of the physics COP, where instructors (I) present the reasons for using computation (rectangles). While the instructors see and promote many reasons, each student prioritizes a subset of reasons (speech boxes) based on their location and trajectory. We expect that, as these students navigate toward the center of the COP through further coursework and research experiences, they will develop the same holistic understanding as their instructors.

We believe this work offers valuable feedback for these courses and similar efforts in the physics community: It is possible for students to develop realistic perceptions about computation after a single semester, and these perceptions develop uniquely along the students' varied trajectories. Exploring these outcomes further can help physics educators motivate students to engage in computation and optimize the placement of computation in the curriculum. 
[1] S. Wolfram, A new kind of science, Vol. 5 (Wolfram media Champaign, IL, 2002).

[2] N. Chonacky and D. Winch, Am. Journ. Phys. 76, 327 (2008).

[3] C. J. Burke and T. J. Atherton, Am. Journ. Phys. 85, 301 (2017).

[4] R. F. Martin, Journn Phys. Conf. Ser. 759, 012005 (2016).

[5] A. L. Graves and A. D. Light, Comp. Sci. Eng. 22, 50 (2020).

[6] T. J. Bing and E. F. Redish, Am. Journ. Phys. 76, 418 (2008).

[7] M. D. Caballero and S. J. Pollock, Am. Journ. Phys. 82, 231 (2014).

[8] C. E. Wieman, K. K. Perkins, and W. K. Adams, Am. Journ. Phys. 76, 393 (2008).

[9] C. Singh, Am. Journ. Phys. 76, 400 (2008).

[10] O. P. Sand, T. O. B. Odden, C. Lindstrøm, and M. Caballero, PER Conference (2018).

[11] A. Buffler, S. Pillay, F. Lubben, and R. Fearick, Am. Journ. Phys. 76, 431 (2008).

[12] M. J. Obsniuk, P. Irving, and M. Caballero, PER Conference (2015).

[13] D. P. Weller, K. Hinko, and V. Sawtelle, PER Conference (2018).

[14] R. M. Serbanescu, P. J. Kushner, and S. Stanley, Am. Journ. Phys. 79, 919 (2011).

[15] R. Chabay and B. Sherwood, Am. Journ. Phys. 76, 307 (2008).

[16] N. T. Young, G. Allen, J. M. Aiken, R. Henderson, and M. D. Caballero, Phys. Rev. Phys. Educ. Res. 15, 010114 (2019).

[17] M. D. Caballero and L. Merner, Phys. Rev. Phys. Educ. Res. 14, 020129 (2018).

[18] B. R. Lunk and R. Beichner, PER Conference 2016, PER Conference, 208 (2016).

[19] A. Leary, P. Irving, and M. Caballero, PER Conference (2018).

[20] Y. Joglekar, G. Vemuri, and A. Gavrin, Bulletin of the American Physical Society 65 (2020).

[21] D. P. Weller, and T. E. Bott, M. D. Caballero, and P. W. Irving
arXiv 2105.07981 (2021)

[22] M. D. Caballero, M. A. Kohlmyer, and M. F. Schatz, Phys. Rev. ST Phys. Educ. Res. 8, 020106 (2012).

[23] E. Wenger, Communities of practice: Learning, meaning, and identity (Cambridge university press, 1999).

[24] E. Wenger, Organization 7, 225 (2000).

[25] E. Wenger, R. A. McDermott, and W. Snyder, Cultivating communities of practice: A guide to managing knowledge (Harvard business press, 2002).

[26] G. M. Quan, C. Turpen, and A. Elby, Phys. Rev. Phys. Educ. Res. 14, 020124 (2018).

[27] K. Thornton, S. Nola, R. E. Garcia, M. Asta, and G. B. Olson, JOM 61, 12 (2009).

[28] M. Caballero, PER Conference (2015).

[29] P. W. Irving and E. C. Sayre, Phys. Rev. ST Phys. Educ. Res. 11, 020120 (2015).

[30] D. Weintrop, E. Beheshti, M. Horn, K. Orton, K. Jona, L. Trouille, and U. Wilensky, Journ. Sci. Educ. Tech. 25, 1 (2016).

[31] P. W. Irving, D. McPadden, and M. D. Caballero, Phys. Rev. Phys. Educ. Res. 16, 020143 (2020).

[32] M. Denscombe, Journ. Mixed Meth. Res. 2, 270 (2008).

[33] E. W. Close, J. Conn, and H. G. Close, Phys. Rev. Phys. Educ. Res. 12, 010109 (2016).

[34] http://gopicup.org

[35] D. P. Oleynik and P. Irving, PER Conference (2019).

[36] B. R. Lunk, Ph.D. Dissertation, North Carolina State University (2012).

[37] S. Weatherford and R. Chabay, PER Conference (2012).

[38] S. Weatherford, Ph.D. Dissertation, North Carolina State University (2011).

[39] P. W. Irving and E. C. Sayre, Phys. Rev. ST Phys. Educ. Res. 10, 010109 (2014).

[40] S. J. Taylor and R. Bogdan, Introduction to qualitative research methods: The search for meanings (Wiley Interscience, 1984). 